Association for Information Systems

AIS Electronic Library (AISeL)

Wirtschaftsinformatik Proceedings 2005

Wirtschaftsinformatik

February 2005

\title{
Ableitung von IT-Strategien durch Bestimmung der notwendigen Wandlungsfähigkeit von Informationssystemarchitekturen
}

Katja Andresen
Universität Potsdam
Norbert Gronau
Universität Potsdam
Simone Schmid
Universität Potsdam

Follow this and additional works at: http://aisel.aisnet.org/wi2005

\section{Recommended Citation}

Andresen, Katja; Gronau, Norbert; and Schmid, Simone, "Ableitung von IT-Strategien durch Bestimmung der notwendigen Wandlungsfähigkeit von Informationssystemarchitekturen" (2005). Wirtschaftsinformatik Proceedings 2005. 4.

http://aisel.aisnet.org/wi2005/4

This material is brought to you by the Wirtschaftsinformatik at AIS Electronic Library (AISeL). It has been accepted for inclusion in Wirtschaftsinformatik Proceedings 2005 by an authorized administrator of AIS Electronic Library (AISeL). For more information, please contact elibrary@aisnet.org. 
In: Ferstl, Otto K, u.a. (Hg) 2005. Wirtschaftsinformatik 2005: eEconomy, eGovernment, eSociety; 7. Internationale Tagung Wirtschaftsinformatik 2005. Heidelberg: Physica-Verlag

ISBN: 3-7908-1574-8

(C) Physica-Verlag Heidelberg 2005 


\title{
Ableitung von IT-Strategien durch Bestimmung der notwendigen Wandlungsfähigkeit von Informationssystemarchitekturen
}

\author{
Katja Andresen, Norbert Gronau, Simone Schmid \\ Universität Potsdam
}

Zusammenfassung: Wandlungsfähigkeit von Informationssystemen ist zu einem wesentlichen Wettbewerbsfaktor geworden. Die derzeit unzureichende methodische Unterstützung zur Umsetzung von Wandlungsfähigkeit führt in Unternehmen häufig zu ungenutzten Potenzialen einer leistungsfähigen Struktur durch die eingesetzte Informationstechnologie.

Ziel des Forschungsprojektes CHANGE ist es, Methoden und Vorgehensmodelle $z u$ entwickeln, die eine dauerhafte Wandlungsfähigkeit von Informationssystemen unterstützen. Dazu wird im Rahmen dieses Beitrages ein Verfahren vorgestellt, welches der Forderung zur Ermittlung der notwendigen Wandlungsfähigkeit unter Einbeziehung des Unternehmensumfeldes nachkommt. Als wesentliches Ergebnis wird ein Kennzahlensystem entwickelt, das zum einen die Umweltsituation als Indikator für den Wandlungsdruck eines Unternehmens beschreibt. Im nächsten Schritt werden Kriterien zur Ermittlung des Wandlungspotentials der eingesetzten IT herangezogen. Abschließend werden beide Dimensionen zusammengeführt und in ihrer Bedeutung für die IT Strategie eines Unternehmens interpretiert.

Schlüsselworte: Wandlungsfähigkeit, Informationssystemarchitektur, Indikatoren, Unternehmenswandel, Umweltturbulenzen

\section{Stand der Forschung}

Das Thema Wandlungsfähigkeit ist ein relativ junges Forschungsgebiet, das zunächst über den Betrachtungsbereich der Fabrikplanung in den Fokus von Industrie und Forschung gerückt ist.

Wandlungsfähigkeit wird in der Fabrikplanung als neues Ziel angesehen. Unter verschiedenen Aspekten wurden Gestaltungsrichtlinien für modulare und wandelbare Fabriken erstellt [NoK102, West02, EvLa ${ }^{+}$02, $\mathrm{WiEr}^{+} 03$ ]. Zu den Wandlungsobjekten mit strategischer Bedeutung gehört auch die Informationssystemarchitektur (ISA) eines Unternehmens. Weiterer Forschungsbedarf besteht darin, Instrumente zur Ermittlung der anforderungsgerechten und wirtschaftlichen Wandlungsfähigkeit zu entwickeln. Zur Ermittlung der notwendigen Wandlungsfähigkeit 
wird die Umwelt eines Unternehmens betrachtet und die Auswirkungen von Umfeldturbulenzen in Beziehung zur Informationssystemarchitektur gesetzt.

Vorarbeiten dazu existieren bereits auf der Prozessebene produzierender Unternehmen [Hart02]. Ein praxistaugliches Verfahren, das Rückschlüsse auf die Ausprägungstiefe einzelner Wandlungsobjekte erlaubt, steht bisher jedoch noch nicht zur Verfügung. Eine methodisch unterstützte Vorgehensweise zur Verbindung von Turbulenzen im Unternehmensumfeld mit der Wandlungsfähigkeit der Informationssystemarchitektur (ISA) ist nicht vorhanden.

\section{Die Wirkung von Umweltturbulenzen auf Informationssystemarchitekturen}

Mit dem Begriff Umweltturbulenzen werden hier die auf das Unternehmen einwirkenden dynamischen Größen des unternehmensspezifischen Umfelds bezeichnet. Treten derartige Umweltveränderungen (Turbulenzen) ein, so ist zu ihrer Bewältigung eine Anpassung der Unternehmensstrukturen notwendig, um die Existenz und den Erfolg der Unternehmung sicherzustellen [Pfau97, S. 1]. Insbesondere die eingesetzten Informationssysteme als integrale Bestandteile des Unternehmens liefern einen essenziellen Beitrag zur Wandlungsfähigkeit [LeHu98].

\subsection{Die Problemstellung}

Infolge der turbulenten Umwelt von Unternehmen ist der Unternehmenswandel zu einem Dauerzustand avanciert [Krüg98, S. 228 ff]. Dies betrifft alle Bereiche des Unternehmens - insbesondere Geschäftsprozesse, Strukturen und die zunehmende Informationsdichte und-technologie.

Produktlebenszyklen werden deutlich kürzer, dies führt zu häufigen Veränderungen der sie begleitenden Geschäftsprozesse. Neue Technologien wie das Internet, EAI (Enterprise Application Integration) oder Web Services verändern die Gestaltung der Wertschöpfungsprozesse, die Integration mit Lieferanten und die Kommunikation mit Kunden [FrSt02, ScKi03]. Ebenfalls berücksichtigt werden müssen neue Managementmethoden, organisatorische und rechtliche Veränderungen [Pico03, S. 2 ff., KiWa03, S. 410 ff].

In einem turbulenten Umfeld mit zunehmend kürzer werdenden Turbulenzintervallen ist es daher notwendig, die einzelnen Unternehmensbestandteile auf den Wandel auszurichten. Die Auswirkungen des Wandels lassen sich unter den Stichworten „Verknappung der Ressource Zeit“, „Verknappung der Ressource Kapital“" und „Steigerung der Komplexität" zusammenfassen [DoLa02]. Die auf den Unternehmen lastenden Anforderungen zum Wandel können in der Praxis oft nicht zeitgerecht und effizient umgesetzt werden. Gründe hierfür liegen in der 
verzögerten Erkennung von Umweltturbulenzen, insbesondere aber auch in strukturellen Wandlungshemmern bei Informationssystemen und Organisation.

Zur Beherrschung der Turbulenz ist es erforderlich, die Informationssystemarchitektur (ISA) wandlungsfähig zu gestalten. Dazu wird in diesem Beitrag ein Verfahren zur Bestimmung der Wandlungsfähigkeit von ISA vorgestellt, das die unternehmensexternen Rahmenbedingungen und die in der gegebenen ISA bereits vorhandenen Potenziale der Wandlungsfähigkeit berücksichtigt. In den folgenden Abschnitten wird zunächst das zugrunde liegende Verständnis von Wandlungsfähigkeit erläutert und danach ein Verfahren zur Ermittlung der erforderlichen Wandlungsfähigkeit von ISA vorgestellt.

Hierfür werden zunächst diejenigen externen Turbulenzgrößen erfasst, die maßgeblichen Einfluss auf das Unternehmen und seine kostengetriebenen IT- Anwendungslandschaft nehmen. Bezogen auf die Wertschöpfungskette des Unternehmens zeichnet sich eine homogenisierte, stark integrierte und brachenorientierte Ausrichtung im Anwendungsbereich ab [StPe04].

Um eventuelle Defizite der Wandlungsfähigkeit auf Seiten der ISA aufzudecken, werden geeignete Indikatoren bestimmt, die Aufschluss über die Wandlungsfähigkeit der Informationssystemarchitektur geben. Durch Gegenüberstellung von Turbulenzanforderungen und vorhandenem Potenzial an Wandlungsfähigkeit werden Handlungsempfehlungen für die Weiterentwicklung der Informationssystemarchitektur abgeleitet.

\subsection{Der Begriff der Wandlungsfähigkeit}

Im Kontext des Unternehmens bezeichnet Wandlungsfähigkeit bzw. Agilität die Forderung nach wirtschaftlichem Handeln der Akteure unter stetig wechselnden und unvorhersehbaren Rahmenbedingungen [Gold95, S. 3].

Wandlungsfähigkeit lässt sich definieren als die Fähigkeit eines Systems, sich selbst effizient und schnell an veränderte Anforderungen anpassen zu können. Von der Flexibilität und der Adaptivität kann die Wandlungsfähigkeit durch die Fragen „Wer erkennt den Änderungsbedarf?“ und „Wer entwickelt geeignete Alternativen?" abgegrenzt werden.

Flexibilität wird die Eigenschaft eines Systems genannt, das einem Änderungsbedarf ein entsprechendes aktivierbares Änderungspotenzial im System gegenüberstellt [Knof92, S. 67]. Bei der Flexibilität reicht es aus, wenn die Erkennung von Veränderungsbedarf und die Verfügbarmachung von Änderungen von außen kommen.

Bei der Adaptivität erkennt das System den Änderungsbedarf selbst (etwa bei Lernsystemen), geeignete Alternativen werden aber von außen bereitgestellt. 


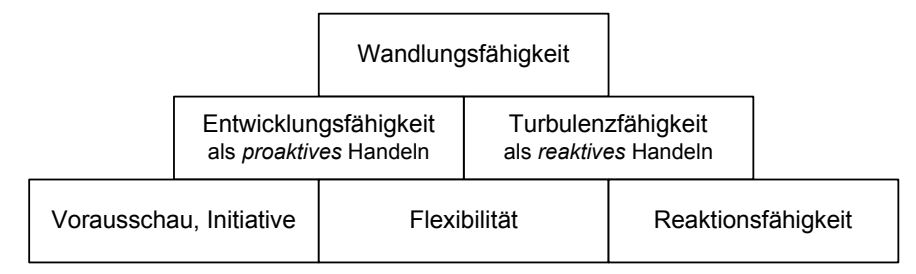

Abbildung 1: Bausteine der Wandlungsfähigkeit nach Spath [Spat+03, S. 9]

Um den verschiedenen Zeithorizonten und Veränderungsintensitäten der Einflüsse aus einer turbulenten Umwelt gerecht zu werden, müssen neben reaktiven auch proaktive Fähigkeiten etabliert werden (Abbildung 1), damit Wandlungsfähigkeit vorliegt [Spat ${ }^{+}$03, S. 9ff]. Wandlungsfähigkeit führt dazu, dass nicht nur Veränderungsbedarf erkannt, sondern auch Alternativen zu seiner Befriedigung aus dem System heraus selbst generiert werden.

Die Entwicklungsfähigkeit bezeichnet dabei die proaktive Fähigkeit des Unternehmens zur nachhaltigen Gestaltung der Unternehmensstrukturen bei längerfristig prognostizierbaren wechselnden Anforderungen. Die Turbulenzfähigkeit hingegen erfordert Reaktionsfähigkeit, um auf die unvorhersehbaren Umweltveränderungen angemessen reagieren zu können [Spat ${ }^{+} 03, \mathrm{~S}$. 10].

\subsection{Informationssystemarchitekturen}

In diesem Abschnitt wird zuerst auf verschiedene Definitionen der Informationssystemarchitektur eingegangen, bevor die Architekturauffassung der Autoren verdeutlicht wird.

Der Begriff Architektur kann allgemein als eine abstrakte, ganzheitliche Betrachtung von Strukturen und Mustern mit Planungscharakter aufgefasst werden $\left[\mathrm{Bas}^{+}\right.$03, S. 19 ff]. Architekturen sind in der Regel das Ergebnis eines Planungsprozesses und stellen nach ihrer Definition selbst einen Masterplan bzw. Bauplan für ganzheitliche Realisierungen zukünftiger Maßnahmen dar. Im Zusammenhang mit Informationssystemen wird dieser Generalbebauungsplan auch als Informationssystemarchitektur bezeichnet [Krcm90, S. 398].

Nach einem Modell von SINZ besteht die Architektur der Informationssysteme aus unterschiedlichen Modellebenen, die unter einem bestimmten Blickwinkel eine vollständige Beschreibung eines Informationssystems vornehmen. Dieser Blickwinkel kann z.B. datenorientiert, prozessorientiert oder objektorientiert sein. Ein Metamodell legt für jede Modellebene ein entsprechendes Modellierungskonzept fest [Sinz 2002, S. 1056].

LAUDON beschreibt die ISA als spezielle Anordnung der unternehmensweiten IT, die im Einklang mit der Organisation steht, um sowohl kurzfristig als auch nachhaltig bestimmte Unternehmensziele zu erreichen [Laud02, S. 26]. 
In diesem Beitrag wird unter einer ISA das Zusammenwirken technologischer, organisatorischer und psychosozialer Aspekte bei der Entwicklung und Nutzung von betrieblichen soziotechnischen Informationssystemen verstanden. Informationssystemarchitekturen stellen dabei eine Sammlung von Vorgehensweisen, Methoden und Elementen zur Planung, Realisierung und Nutzung betrieblicher Informationssysteme unter Berücksichtigung der komplexen Abhängigkeiten zwischen den einzelnen Ebenen - hier vor allem der Organisation und IT dar (Abbildung 2).

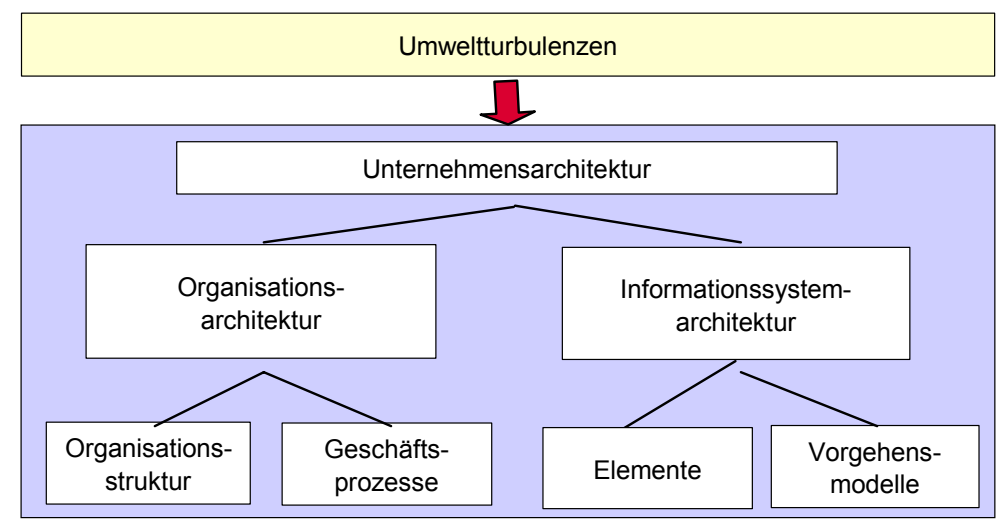

Abbildung 2: Bestandteile des Unternehmens und ihre externen Einflussgrößen

\section{Ein Verfahren zur Ermittlung der notwendigen Wandlungsfähigkeit von Informationssystem- architekturen}

Die Wandlungsfähigkeit von Unternehmen wird immer häufiger als Wettbewerbsfaktor in einem turbulenten Umfeld herausgestellt [AnGr04]. Eine zentrale Herausforderung bei der Erhöhung der Wandlungsfähigkeit von Unternehmen besteht darin, Prozesse effizient zu gestalten, um rechtzeitig und gezielt handeln zu können.

Die Erarbeitung einer Methodik, um die spezifische Bedeutsamkeit der Wandlungsnotwendigkeit für das einzelne Unternehmen ermitteln zu können, bildet die Grundlage für ein strukturiertes Vorgehen zur Anpassung der Wandlungsfähigkeit eines Unternehmens in dynamischen und sprunghaften Entwicklungen des Umfeldes.

Es wird beispielhaft eine Methode vorgestellt, die es Unternehmen ermöglichen soll, anhand der für das Unternehmen relevanten Umweltfaktoren die Anfälligkeit des Unternehmens gegenüber äußeren Einflüssen zu spezifizieren. Dieser Betrach- 
tung wird zugrunde gelegt, dass eine erhöhte Anfälligkeit dann besteht, wenn Umwelteinflüsse als turbulent eingeschätzt werden. Somit besteht für das Unternehmen ein größerer Veränderungsdruck. Im Gegenzug bedeuten als konstant eingeschätzte Umweltbedingungen für eine Unternehmensorganisation, dass keine Notwendigkeit besteht, Veränderungen zu antizipieren

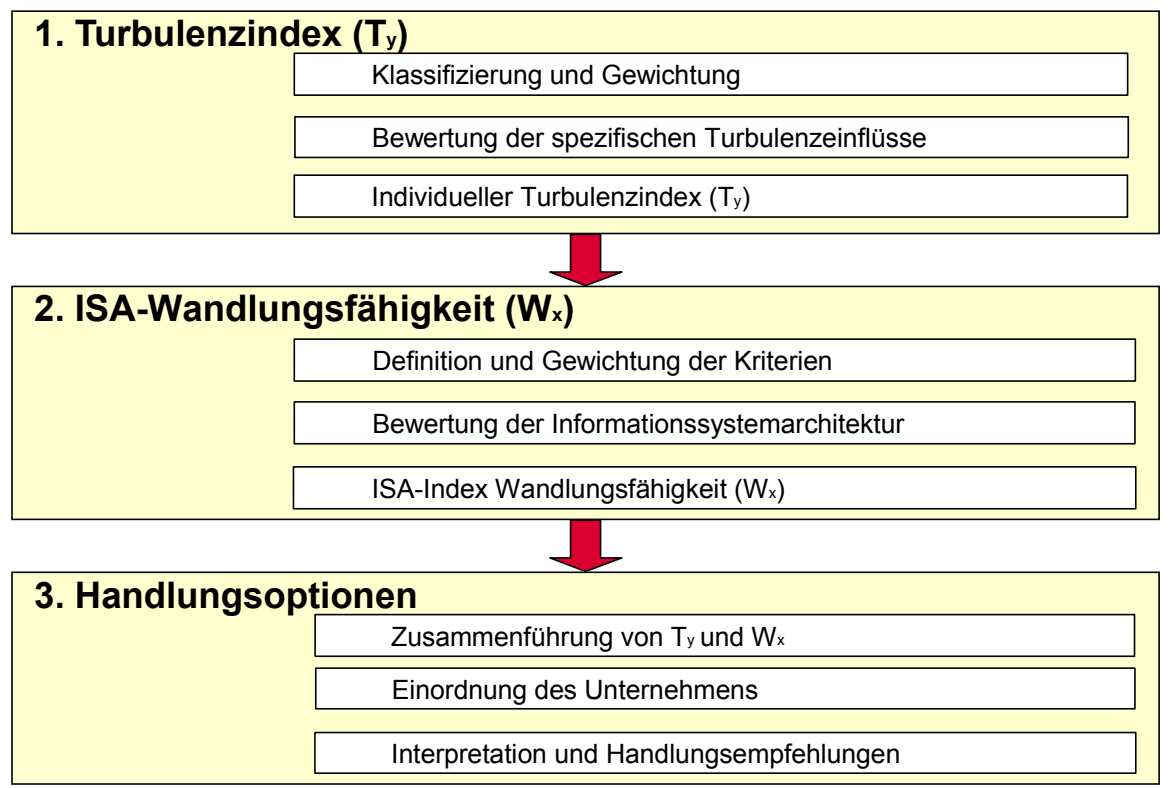

Abbildung 3: Vorgehen zur Ermittlung von Handlungsempfehlungen

Die hier vorgestellte Methodik wurde im Rahmen des vom BMBF geförderten Projektes CHANGE - Wandlungsfähige Auftragsabwicklungssysteme ${ }^{1}$ entwickelt. Sie beruht auf den Prinzipien der branchenbezogenen Klassifizierung von Unternehmen und Umweltfaktoren. In Phase 1 erfolgt die Analyse unabhängig von den spezifischen Gegebenheiten des einzelnen Unternehmens, gerichtet auf generelle Einflussfaktoren in einem bestimmten Industriezweig. In einem zweiten Schritt wird die Sensibilität des einzelnen Unternehmens berücksichtigt. Aus dieser Bewertung wird anschließend eine Kennziffer generiert, die den Turbulenzindex (Ty) eines Unternehmens darstellt.

In der zweiten Phase wird die Informationssystemarchitektur des Unternehmens auf Wandlungsfähigkeit hin untersucht. Die sieben wichtigsten und voneinander unabhängigen Indikatoren für die Wandlungsfähigkeit von Informationssystemarchitekturen werden auf Vorhandensein in den verwendeten, unternehmensindividuellen Anwendungen überprüft. Alle Bewertungen der Teilbereiche bilden dann den aggregierten Index für die Wandlungsfähigkeit des Unternehmens (Wx).

1 www.change-projekt.de, Förderkennzeichen BMBF 01 ISC20 F 
In Phase 3 werden beide Indizes in einem Portfolio zusammengeführt und einem von vier Quadranten zugeordnet. Aus der so gefundenen Position eines Unternehmens lassen sich Handlungsempfehlungen für die zukünftige Ausgestaltung der IT-Strategie ableiten.

Da das Verfahren individuelle Relevanzwerte des Unternehmens miteinbezieht ist eine Übertragbarkeit auf beliebige Unternehmen möglich. Die branchenspezifischen Informationen können aus vorliegendem statistischem Zahlenmaterial generiert werden. Für die unternehmensindividuellen Bereiche sind detaillierte Informationen, die sich zum Beispiel über Interviews gewinnen lassen, notwendig.

\section{Bestimmung und Bewertung der Turbulenz- kriterien der Unternehmensumwelt}

Im Kontext dieses Beitrages wird die systemtheoretische Betrachtungsweise verfolgt. Unternehmen werden als evolvierende Systeme zur Bewältigung komplexer Aufgabenstellungen in einer turbulenten Umwelt begriffen [Thom67, ReMö99].

Die Unsicherheit der Märkte als Charakteristikum des zweckbezogenen Kontexts sind hier von zentraler Bedeutung zur Bestimmung der reaktiven Fähigkeiten des Systems Unternehmen [ReMö99]. Turbulenzkriterien werden als äußere Einflüsse aufgefasst, die eine Veränderung am Zustand des Systems Unternehmen bewirken. Grundlegende Funktion jedes Systems ist die Bestandssicherung - die Anpassung an die Erfordernisse der umgebenden Umwelt.

Ein System muss, um handeln zu können, ein Abbild von seiner Umwelt besitzen und handlungsrelevante Teile und Aspekte seiner Umwelt identifizieren [DrWe84]. Dafür gibt es in der Organisationstheorie eine Vielzahl von Konzepten. Allen Ansätzen ist gemein, die Umwelteinflüsse auf eine Organisation in Gruppen zu klassifizieren. Dazu wurde eine große Zahl von Schemata entwickelt. Auf der inhaltlichen Ebene findet eine Einteilung in zwei Zonen statt, die Aufgabenumwelt sowie die globale (generelle) Umwelt [StSc00].

Die Aufgabenumwelt charakterisiert jene Faktoren, mit denen die Unternehmensorganisation in direkter Interaktion steht. Sie wird als direktes Wettbewerbsumfeld verstanden. Die den Gesamteinfluss bildenden Umweltfaktoren werden in Form von Teilklassen beschrieben. Folgende Faktoren [Port84] gelten als Determinanten des Aufgabenumfeldes. Fünf Wettbewerbskräfte sind gemeinsam bestimmend für die Situation einer Branche:

- Rivalität unter den bestehenden Anbietern (Wettbewerbsfaktor)

- Potentielle Neuanbieter (Konkurrenzfaktor)

- Substitutionsprodukte (Ersatzfaktor)

- Abnehmer (Verhandlungsfaktor) 
- Lieferanten (Verhandlungsfaktor)

Mit der globalen Umwelt werden alle Kräfte und Faktoren bezeichnet, die nicht Bestandteil des Systems sind. Anhand von Faktorkatalogen [Hall72; FaNa86; MüLe03] wurden potenziell relevante Einflussfaktoren ermittelt, die sich in folgende Teilbereiche untergliedern:

- Technologische Umwelt

- Politisch-rechtliche Umwelt

- Arbeitsmarkt

- Geld- und Kapitalmarkt

- Ökologische Umwelt

Daraus resultieren insgesamt zehn Umweltfaktoren mit Systembezug. Sie bilden die Relevanzbereiche der Umwelt der Unternehmensorganisation. Für die Charakterisierung des Umfeldes einer konkreten Organisation stellt die Auflistung von Umweltfaktoren einen ersten Schritt dar.

\section{Das Verfahren}

Ausgangspunkt für die Definition der Turbulenzkriterien ist die durchgängige Abbildung der zu betrachtenden Umweltturbulenzen. Im Spektrum zwischen Unternehmen und Markt existiert eine Vielzahl alternativer Reaktionsmuster von Unternehmensorganisationen auf vorherrschende Umwelteinflüsse. Die Filterung der systemkritischen Umwelteinflüsse erfolgt über die unterschiedliche Gewichtung der Turbulenzfaktoren, welche innerhalb der gewählten Methodik branchenspezifisch und somit normativ gebildet werden. Die Festlegung der Gewichtungen ist ein $\mathrm{Ma}$ für die allgemeine Relevanz eines Umweltfaktors auf eine Klasse von Organisationseinheiten. Im nächsten Schritt wird die Sensibilität der individuellen Unternehmensorganisation auf die zu betrachtende Turbulenz bewertet. Ein unternehmensspezifischer Faktor ist dafür das Maß, das die Wahrscheinlichkeit des Eintretens einer realisierten Turbulenz der Umwelt beschreibt, wobei vom IstZustand ausgegangen wird. Durch die Abbildung der Faktoren vergleichbarer Unternehmen lassen sich daraufhin qualitative Aussagen über die Flexibilisierungsnotwendigkeiten gegenüber einzelner Teilklassen von Umwelteinflüssen erkennen und kritische Turbulenzen identifizieren. Tabelle 1 zeigt die Ermittlung des spezifischen Turbulenzindex am Beispiel eines mittelständischen Kabelherstellers.

Durch die Anwendung des zu Grunde liegenden Modells ist es möglich, die Anfälligkeit des Unternehmens in den zuvor erfassten Turbulenzklassen in einem ersten Unternehmensindex (Turbulenzindex) zusammenzuführen. Sodann ist eine allgemeine Aussage über die aggregierte Anfälligkeit gegenüber Umweltturbulenzen möglich.

Die verwendete Methodik zeigt, dass auf den unterschiedlichen Analyseebenen im Wesentlichen die gleichen Bereiche betrachtet werden, die ein Modell des Unternehmens und seiner Einbettung in den Markt darstellt. Die Untersuchungsperspek- 
tive ist einerseits auf generelle Einflüsse gerichtet; zum anderen auf unternehmensindividuelle Aspekte.

\begin{tabular}{lrrr}
\multicolumn{1}{c}{ Faktor } & Gewicht & Wirkung & Punkte \\
& 100 & $0<=\mathrm{p}<=10$ & 90 \\
Rivalität & 12 & 7,5 & 50 \\
Neuanbieter & 10 & 5 & 58,5 \\
Substitutionsprodukte & 13 & 4,5 & 337,5 \\
Abnehmer & 45 & 7,5 & 52,5 \\
Lieferanten & 7 & 7,5 & 32,5 \\
Technologische Umwelt & 5 & 6,5 & 5 \\
Politisch-rechtliche Umwelt & 2 & 2,5 & 6 \\
Arbeitsmarkt & 2 & 3 & 12 \\
Geld- und Kapitalmarkt & 2 & 6 & 6 \\
Ökologische Umwelt & 2 & 3 & $\mathbf{6 5}$ \\
Turbulenzindex & & &
\end{tabular}

Tabelle 1: Ermittlung des Turbulenzindex Ty

Die Zuordnung eines Unternehmens und die Bewertung einer Branche reflektieren immer den Ist-Zustand zu einem bestimmten Zeitpunkt, in dem alle wesentlichen Strukturen und Bedingungen des Umfeldes erfasst werden.

Ein problematischer Aspekt besteht in der adäquaten Berücksichtigung von wesentlichen Eigenschaften die zu jedem Zeitpunkt vorherrschen. Die Branchenbeschreibung als auch die Unternehmensbewertung muss für jede Einordnung des Unternehmens neu hinterfragt werden [Port98].

\section{Identifizierung der Wandlungsfähigkeit von Informationssystemarchitekturen}

Um die Wandlungsfähigkeit von Informationssystemarchitekturen zu bewerten, muss zunächst eine adäquate Auswahl von Indikatoren bestimmt werden. Ein Indikator stellt hier eine wünschenswerte Eigenschaft eines Bestandteiles einer Informationssystemarchitektur bzw. des Zusammenwirkens der Elemente dar. Die hier erläuterten Indikatoren können im Wesentlichen aus zwei Gebieten abgeleitet werden. In der Fabrikplanung werden seit einiger Zeit für die Ausführung der Gebäude und Fertigungs- bzw. Montagesysteme Gestaltungsparameter aufgeführt, 
die zu wandlungsfähigen Fabriken führen [Hern02]. Diese Faktoren werden in angepasster Form auf Informationssystemarchitekturen übertragen. Zum anderen haben sich autopoietische Systeme wie etwa natürliche Ökosysteme als anpassungsfähig im Sinne der oben gegebenen Definition von Wandlungsfähigkeit erwiesen. Durch Übertragung wesentlicher Eigenschaften autopoietischer Systeme wird versucht, auch Informationssystemarchitekturen wandlungsfähig zu gestalten. Nach Ansicht der Autoren bilden die im Folgenden beschriebenen Indikatoren die Basis für ein geeignetes Beurteilungsinstrument.

\subsection{Kriterien zur Beurteilung der Wandlungsfähigkeit}

Aus der Fabrikplanung stammen die Indikatoren Skalierbarkeit, Modularität, Mobilität und Interoperabilität. Die Betrachtung autopoietischer Systeme fügt die Indikatoren der Selbstorganisation, Selbstähnlichkeit und Redundanz hinzu. Nach Ansicht der Autoren spielt zur Integration dieser Indikatoren insbesondere der Aspekt des Wissens der Mitarbeiter eine entscheidende Rolle. Dieser bildet den achten Indikator.

\section{Skalierbarkeit}

Unter der Skalierbarkeit einer Informationssystemarchitektur ist dessen kapazitätsmäßige Gestaltung zu verstehen [Wall96]. Dieser Indikator fordert eine effiziente Anpassung (nach oben und nach unten!) an veränderte Mengen von zu verarbeitenden Informationen. Dies kann sowohl durch die Software als auch durch die Hardwarearchitektur erreicht werden. Durch das automatische Hinzufügen und Entfernen von Ressourcen wie beispielsweise Speicher oder Rechnerleistung wird ein reibungsloser Systembetrieb sichergestellt. Bezogen auf die Software, hier Anwendungen, müssen diese so konzipiert sein, dass sie bei steigenden Anforderungen gleichmäßig mitwachsen können. Ein Beispiel stellt die von einem ERPSystemhersteller entwickelte gleitende Nutzbarkeit von organisatorischen Gliederungsebenen für die Kostenrechnung dar. Das System stellt stets so viele Ebenen zur Verfügung, wie benötigt werden [Gümb04].

\section{Modularität}

Modularität bzw. Modularisierung bedeutet allgemein die Strukturierung eines Systems in kleine, teilautonome und übersichtliche Subsysteme [Pico ${ }^{+}$03, S.230]. Diese Subsysteme stellen die sog. Module dar. Ein Modul besteht aus einem Modulrumpf und einer Modulschnittstelle. Letzteres beinhaltet eine Spezifikation über die Leistung und die Eigenschaften, die für sein Umfeld von Bedeutung sind. Der Modulrumpf implementiert die in der Schnittstelle spezifizierte Definition über das Modul [AiDo04]. Somit können einzelne Module aufwandsarm entfernt, durch andere ersetzt oder einem anderen System hinzugefügt werden. Damit stellt die Modularität eine Möglichkeit zur effizienten Kombination, Wiederverwendung und der schnellen Änderung von informationstechnischen Anwendungen dar. 
Modularität fordert ein Überdenken des klassischen Client-Server-Paradigmas angesichts der nicht mehr bewältigbaren Komplexität heutiger StandardAnwendungssysteme. Eine mögliche Lösung stellen Peer-to-Peer-Systeme [HBK03, S. 1105ff] oder serviceorientierte Architekturen dar [Hah03, S. 557ff].

\section{Mobilität}

Mobilität hat in diesem Zusammenhang zwei Bedeutungen. Zum einen ist damit der räumlich und zeitlich unbegrenzte Zugriff auf die Anwendungen der Informationssystemarchitektur und somit auf seine Daten und Funktionen mittels verschiedener Technologien gemeint. Beispielsweisen zählen hierzu Web-Browser, Terminal-Server oder ein Virtuelles Privates Netzwerk, mittels dessen auf die Anwendungen und Daten des Informationssystems zugegriffen werden kann. Die andere Dimension erfasst die Plattformunabhängigkeit der Anwendungen. Diese Ungebundenheit umfasst beispielsweise die von der verwendeten Hardware, dem Betriebssystem, den Datenbanksystemen oder den Applikationsservern. Ein Beispiel hierfür ist die Service Oriented Architecture (SOA), welche Services zur Verfügung stellt, die plattformunabhängig miteinander interagieren [Dunn03].

\section{Interoperabilität}

Interoperabilität bezeichnet die Fähigkeit von Anwendungen miteinander zusammenzuarbeiten. Unabhängig von der verwendeten Hardware, den eingesetzten Betriebssystemen, der verwendeten Netzwerktechnologie und der Realisierung einer Anwendung kann eine Kooperation zwischen diesen Anwendungen erfolgen. Interoperabilität erlaubt den einfachen Zugang zu verschiedenen (auch raumbezogenen) Daten- und Verarbeitungsressourcen innerhalb eines Arbeitsablaufs bzw. die einfache Verknüpfung unterschiedlicher Informationssysteme. Durch die Erfordernis der Kooperation mehrerer Anwendungssysteme zur Abbildung der Geschäftsprozesse wird der Interoperabilität zwischen den Informationssystemen eine zentrale Rolle zugeschrieben. Für die Kooperation zwischen Unternehmen bedeutet die Interoperabilität eine erhöhte Kommunikations- und Kooperationsfähigkeit. Das wäre ein wesentlicher Schritt zur Erreichung einer erhöhten Kommunikation, Interaktion und Transaktion zwischen Unternehmen.

Dieser Indikator bezieht sich auf die Fähigkeit von Systemelementen, ein hohes $\mathrm{Ma}$ an Kompatibilität zur Verfügung zu stellen. Beispiele dafür sind ISAElemente, die Standards berücksichtigen. Das wesentliche Ziel der Standardisierung im IT-Bereich besteht darin, den Aufwand für die Entwicklung von Konnektoren und Funktionen bzw. Prozessen zu minimieren. Somit können Systemelemente besser miteinander verbunden werden und die Integrationsfähigkeit von einzubindenden Elementen wird gesteigert.

Die Standardisierung bezieht sich in diesem Beitrag auf die Daten-, Objekt-, und Prozessebene. Standardisierung auf Datenebene bezieht sich insbesondere auf allgemeinverständliche Datenformate wie z.B. XML. Standardisierung auf Objektebene bezieht sich auf die semantische Ebene der Kommunikation zwischen Informationssystemen. QUANTZ/WICHMANN identifizieren in diesem Bereich vier 
wesentliche Gegenstandsbereiche [QuWi03, S. 63ff]. Hierzu zählen die Produktidentifikation, Produktklassifikation und -beschreibung, Katalogaustauschformate und Transaktionen (Austausch von Geschäftsdokumenten). Die Prozessstandards stellen derzeit die höchste Ebene der Standardisierung dar. Hierzu gehören ebXML oder RosettaNet. Die Standardisierung der Prozessebene ist jedoch sehr komplex und viele Bemühungen zur Etablierung sind noch nicht so weit fortgeschritten, da viele Unternehmen sich derzeit anderen Herausforderungen widmen.

Die Einhaltung von Standards zur Planung, Entwicklung und Nutzung von Informationssystemarchitekturen gewährleistet eine bessere inner- und zwischenbetriebliche Integrationsfähigkeit zwischen unterschiedlichen Informationssystemen, eine geringere Fehleranfälligkeit durch stärker verbreitetes Know-how zum Gebrauch der Standards und zur schnelleren Realisierung der Lösung [QuWi03].

\section{Selbstorganisation}

Selbstorganisation (Autopoiesis) bezeichnet die Fähigkeit eines Systems durch selbstregulierende und -lenkende Mechanismen die Systemstruktur aus den Prozessen ihrer eigenen Leistung heraus zu bestimmen, um den langfristigen Systembestand zu gewährleisten [MaVa87]. Die Systemelemente bzw. Subsysteme erzeugen dabei ihre eigene Ordnung, indem sie Informationen über ihre Umwelt und ihre Wechselwirkung mit der Umwelt aufnehmen, diese zu einem „Modell“ verdichten und in der realen Welt gemäß diesem Schema handeln [Gell98].

Da autopoietische Systeme ständig mit der Umwelt in Wechselwirkung stehen, verändern sich die internen Schemata bei einer Modifikation der Umwelt. Die aus dem System resultierende Struktur bzw. Ordnung weist durch das Prinzip der Selbstorganisation eine hohe Leistungsfähigkeit auf [Schr03, S.17]. Diese effizienten adaptiven und zugleich autonomen Systeme sind beispielsweise in der $\mathrm{Na}-$ tur (z.B. Ökosysteme) zu finden. Diese These ist empirisch belegbar, allerdings zunächst nicht in der Wirtschaftsinformatik.

Die Anforderungen der Selbstorganisation von Informationssystemarchitekturen werden erfüllt, wenn sie in der Lage sind, ihre innere Struktur bzw. Architektur ganz oder teilweise selbst zu bestimmen. Ein Beispiel für ein autopoietisches System stellt das Internet dar, dessen Struktur sich allein durch die Zahl der angeschlossenen Server bestimmt. Ausgehend von wenigen Standards (vgl. Neutralität) werden nahezu alle Aufgaben des Internet dezentral ausgeführt.

\section{Selbstähnlichkeit}

Der Unterschied zwischen Ähnlichkeit und Selbstähnlichkeit besteht darin, dass die Ähnlichkeit als Gleichheit im Sinne geeignet gewählter Abstraktion keine Aussage zum Verhalten bei Vergröberung oder Verfeinerung des Betrachtungsausschnittes macht. Die Selbstähnlichkeit hingegen ist eine Eigenschaft, durch Zusammenlegen oder Aufteilen im Wesentlichen immer wieder gleiche Muster auf einer anderen Größenskala zu erhalten. Man spricht in diesem Fall von einer Skaleninvarianz. Selbstähnliche und selbstorganisierende Elemente führen zu einem 
autopoietischen Systemverhalten, das sich positiv auf die Wandlungsfähigkeit von Informationssystemarchitekturen auswirkt [Gron03, S. 213].

Die Selbstähnlichkeit ist ein Phänomen, das vielen natürlichen Objekten (Wolken, Pflanzen, Gebirge etc.) eigen ist. In verschiedenen Größenmaßstäben zeigen sich immer dieselben Grundstrukturen. Viele chaotische Systeme zeigen selbstähnliches Verhalten. Häufig ist diese Selbstähnlichkeit der einzige Zugang zur Analyse komplexer Strukturen oder dynamischer Systeme. Selbstähnlichkeit als Eigenschaft autopoietischer Systeme korrespondiert mit dem Anspruch der Modellierung auf Verkürzung des Gegenstandsbereiches zur Komplexitätsreduzierung. Selbstähnliche Systeme ermöglichen unterschiedlich tiefe Modellierungen, ohne dass ein Modellierungsergebnis auf einer höheren Ebene aufgegeben werden muss und erleichtern so die Modellierung. In der Organisation wird diese Eigenschaft z.B. bei der Bildung von Produktionsfraktalen [Warn93, S. 200] genutzt.

Als Beispiel für die Vorteile von Selbstähnlichkeit kann die leichtere Erlernbarkeit der Bedienung von Anwendungssystemen genannt werden, die auf unterschiedlichen Ebenen und Plattformen basieren, dennoch aber eine immer wiederkehrende Bedienphilosophie aufweisen.

\section{Redundanz}

Redundanz fordert die Verfügbarkeit von ähnlichen Ressourcen auch über die unmittelbar erforderliche Leistungserstellung hinaus. Sie erzeugt Dezentralität, wenn Aufgaben parallel auf mehreren Applikationsservern ausgeführt werden können und die Ergebnisse z.B. durch Replikationsmechanismen abgeglichen werden können. Redundanz fördert unmittelbar die Wandlungsfähigkeit von Informationssystemarchitekturen, zeigt aber auch die Probleme dieses Gestaltungsparadigmas auf: Zusätzliche Ressourcen, deren Bedarf nicht sicher vorhergesagt werden kann, verursachen sehr wohl Kosten, und zwar vom Zeitpunkt ihrer Verfügbarmachung an. Hier ist zu entscheiden, inwieweit das Unternehmen bereit ist, für eine verbesserte Wandlungsfähigkeit auch Kosten in Kauf zu nehmen. Diesen Kosten stehen dann Erträge gegenüber, wenn aufgrund von Umfeldturbulenzen die Wandlungsfähigkeit benötigt wird.

\section{Wissen über die Informationssystemarchitektur}

Wissen über die Informationssystemarchitektur bezeichnet sowohl das personengebundene (stillschweigendes Wissen) als auch das explizite Wissen. Letzteres liegt beispielsweise in Form von schriftlich fixierten Prozessen, Regeln, Richtlinien, Verantwortlichkeiten, Kommunikationskanälen, Modellen usw. vor. Stillschweigendes Wissen bezieht sich im Zusammenhang dieses Beitrags insbesondere auf die Fähigkeiten der unternehmensinternen Mitarbeiter, die mit der Gestaltung oder Nutzung der ISA befasst sind. Ihre Erfahrungen umfassen beispielsweise das Potenzial, neue Anwendungen zu integrieren, neue Prozesse abzubilden oder neue Wertschöpfungspartner in die Leistungserstellung einzubeziehen. 


\subsection{Anwendung des Indikatorensystems}

Für die oben beschriebenen Indikatoren wird nun für alle betrachteten Elemente der Informationssystemarchitektur der Grad an Übereinstimmung der Eigenschaft des Elements mit dem Indikator ermittelt. Dazu wird eine Skala von 0 bis 100 benutzt, auf der ein Wert von 0 bedeutet, dass der Indikator überhaupt nicht erreicht wird, während ein Wert von 100 bedeutet, dass eine vollständige Übereinstimmung erzielt wurde.

Die einzelnen Elemente der ISA werden auf ihren Beitrag zur Gesamtheit hin gewichtet und der je ISA errechnete Wert mit dem Gewicht des Elements multipliziert. Die so erreichten Punktwerte aller ISA-Elemente werden addiert.

Das Verfahren strebt keinerlei mathematische Genauigkeit an. Es kommt ausschließlich darauf an, eine grobe Einschätzung der Wandlungsfähigkeit der ISAElemente zu erzielen. Obwohl die errechneten Ergebnisse ein gewisses Maß an Genauigkeit suggerieren, ist aufgrund der unscharfen Ermittlungsvorschriften lediglich die Feststellung möglich, ob sich die betrachtete ISA eher im Bereich niedriger oder hoher Wandlungsfähigkeit befindet. In Tabelle 2 findet sich ein Beispiel für das ERP-System eines mittelständischen Kabelherstellers.

\begin{tabular}{lr}
\multicolumn{1}{c}{ Indikatorgruppe } & $\begin{array}{c}\text { Wert } \\
\text { max. } 100\end{array}$ \\
Skalierbarkeit & 20 \\
Modularität & 10 \\
Mobilität & 0 \\
Interoperabilität & 40 \\
Selbstorganisation & 0 \\
Selbstähnlichkeit & 10 \\
Redundanz & 50 \\
Wissen über die ISA & 20 \\
Summe / Zahl der Indikatoren & $\mathbf{1 8 , 7 5}$
\end{tabular}

Tabelle 2: Ermittlung der Wandlungsfähigkeit der ISA am Beispiel eines ERP-Systems

Das Beispiel in Tabelle 2 zeigt, dass das eingesetzte ERP-System sich durch eine geringe Wandlungsfähigkeit auszeichnet. 


\section{Ableitung von Situation und IT-Strategie}

Nachdem die relevanten Aspekte in der Analyse der Ausgangssituation des Untenehmens erhoben und bewertet wurden, erfolgt die Überführung der Kennzahlen in ein Koordinatensystem. Die Portfolio-Darstellung veranschaulicht das Ergebnis der beiden Dimensionen ISA Wandlungsfähigkeit (Wx) und auf der Ordinate den Turbulenzindex (Ty). Als Beitrag zur Priorisierung der Ausrichtung der ITStrategie wurde auf der Grundlage der beiden Einflussmatrizen ein Handlungsportfolio entwickelt. Die Stärke des Einflusses der Umweltturbulenzen (niedrig, hoch) wird in einem vernetzten Zusammenhang dem Grad der ISA Wandlungsfähigkeit (niedrig, hoch) gegenübergestellt. Daraus resultieren vier Zonen, in die ein Unternehmen eingeordnet werden kann, wenn die Ergebnisse in Form von Punktwerten in das Portfolio-Diagramm eingetragen werden (Abbildung 4).

Die Neutrale Zone beschreibt den Zustand geringer Umweltturbulenzen und geringer vorherrschender Wandlungsfähigkeit. Die Transferzone kennzeichnet ein Unternehmen, welches über einen hohen Grad an Wandlungsfähigkeit in seiner ISA bei geringen Umweltturbulenzen verfügt. In der Kritischen Zone sind die Umweltturbulenzen stark ausgeprägt, während die ISA kaum wandlungsfähig ist (Ty $>>$ Wx). In der Idealen Zone sind Umweltturbulenzen und Wandlungsfähigkeit maximal ausgeprägt.

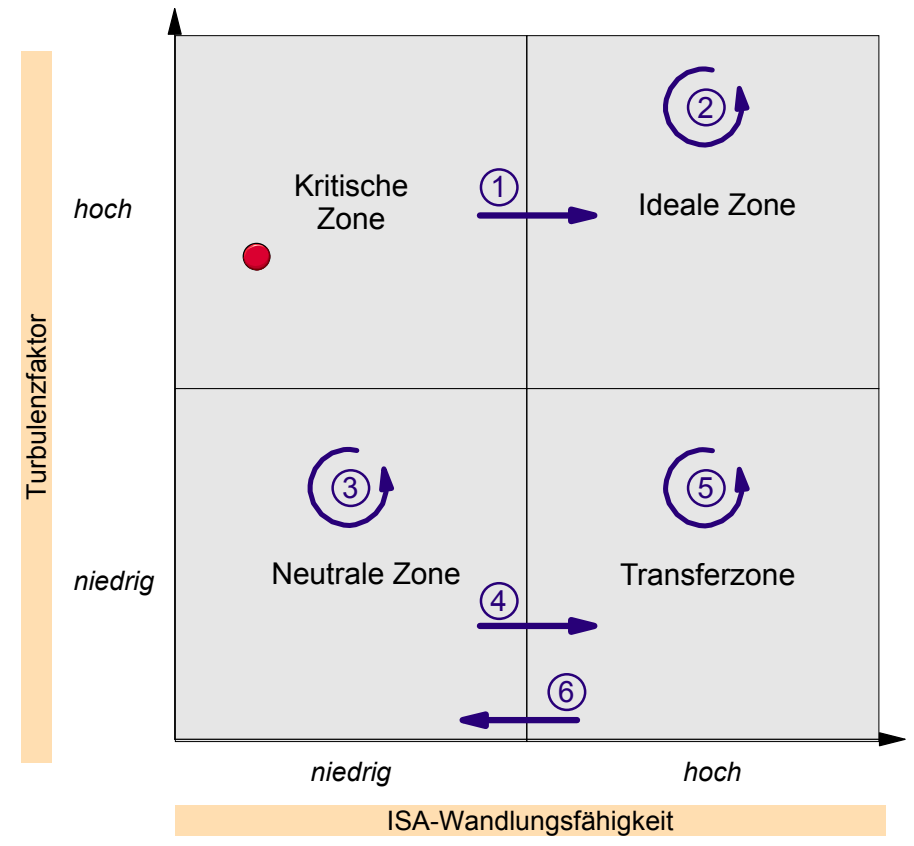

Abbildung 4: Ableitung einer IT Strategie aus dem Portfolio 
Mit den erarbeiteten Daten wird aus dem Ist- das Ideal-Portfolio entwickelt. Die Pfeile verdeutlichen die weitere Entwicklung und erlauben die Ableitung der zu ergreifenden IT Strategie (1 - 6).

1. Wird der Zustand eines Unternehmens durch die Kritische Zone reflektiert, muss die Wandlungsfähigkeit der ISA schnell erhöht werden, damit den Umweltturbulenzen zeitnah adäquat begegnet werden kann. Gelingt es hier, die notwendige Wandlungsfähigkeit umzusetzen, wird das Unternehmen in die Lage versetzt, die Markt- bzw. Umfelddynamik zu beherrschen und darüber hinaus gezielt Turbulenzen zu erzeugen. Das entspricht dem Übergang von der kritischen in die ideale Zone.

2. Das Unternehmen befindet sich in der Idealen Zone: Die Prämisse liegt in der langfristigen Erhaltung der Wandlungsfähigkeit. Ein regelmäßiges Prüfen der Situation anhand der definierten Kriterien für Wandlungsfähigkeit verhindert ein unbemerktes Abweichen des Unternehmens vom Idealkurs und legt die Grundlage für eine mögliche Anpassung, wenn nötig.

3. Bleiben Unternehmen im Umfeld mit geringer Dynamik ausreichend unterstützt von Informationssystemen mit geringer Wandlungsfähigkeit und sind somit in der Neutralen Zone zu finden, steht im Fokus der IT-Strategie des Unternehmens die Weiterentwicklung unter dem Aspekt der Kostenreduktion.

4. Ein vorsichtiger Ausbau der Wandlungsfähigkeit im Rahmen einer bereits definierten IT Strategie kann von einem Übergang von der Neutralen in die Transferzone begleitet werden.

5. Ist die Situation derart, dass die Wandlungsfähigkeit der IT Systeme die Anforderungen des dynamischen Unternehmensumfeldes übererfüllt $(\mathrm{Wi}>>\mathrm{Tj}$ ), ist die Effizienz der eingesetzten ISA in den Vordergrund der IT-Strategie zu stellen, da die Implementierung von Wandlungsfähigkeit immer ein Kostenfaktor ist [HeHo04]. IT-strategisch kann Potenzial zur Kostenreduktion, ohne den Grad der Wandlungsfähigkeit aufzugeben, eine Option sein.

6. Befindet sich das Unternehmen in der Transferzone, ist es ebenfalls möglich, die internen Konsequenzen für den Leistungserstellungsprozess bei einer veränderten IT sind in Bezug auf die Verringerung der Wandlungsfähigkeit zu prüfen. Unter strategischen Gesichtspunkten einer Risikominimierung ist jedoch eine Optimierung der Wandlungsfähigkeit zu bevorzugen, um proaktiv auf Veränderungen eingestellt zu sein. Die Anpassung der IT an die aktuelle Turbulenzlage entspricht demnach einer unter Kosten- und Effizienzgesichtspunkten zu bewertenden Minimalanforderung. 


\section{Zusammenfassung und Ausblick}

Im vorliegenden Artikel wurde ein Verfahren zur Bestimmung der notwendigen Wandlungsfähigkeit im Unternehmen vorgestellt, das sich nicht nur einseitig auf die Abbildung der internen Faktoren beschränkt, sondern neben den Kriterien der Wandlungsfähigkeit auch die Kriterien der einwirkenden Umweltturbulenzen berücksichtigt. Sowohl die internen als auch diese externen Faktoren wurden jeweils einzeln bewertet und konnten durch das Aufstellen einer Gesamtmatrix in Bezug zueinander gebracht und somit im Gesamtprozess effektiv berücksichtigt werden. Dadurch ist es möglich geworden, den individuellen Bedarf an Wandlungsfähigkeit einer spezifischen Unternehmung zu bestimmen und so gegebenenfalls die entsprechenden Maßnahmen für notwendige Veränderungen im Bereich der Informationssystemarchitektur rechtzeitig einzuleiten.

Das Verfahren stellt eine wichtige Hilfe zur Bewältigung des dynamischen Umfeldes der Unternehmung dar. Im Zusammenhang mit einer künftigen Weiterentwicklung des Verfahrens stellt sich hier die Frage nach einer weiteren Verfeinerung bzw. Ergänzung der Kriterien sowohl im Bezug auf die Umweltturbulenzen als auch im Bereich der internen Faktoren. Dabei müssen vier Voraussetzungen gewährleistet werden: Unabhängigkeit, Vollständigkeit, Zweckmäßigkeit und Messbarkeit.

Die einzige zulässige Abhängigkeit, die das Verfahren erlaubt, besteht darin, dass die Kriterien entscheiden, inwieweit das Unternehmen wandlungsfähig ist oder nicht. Ansonsten dürfen unter keinen Umständen weitere Abhängigkeiten bestehen. Hier kann es zu Problemen bei der Abgrenzung der Kriterien zueinander kommen, was eine genaue Einzelüberprüfung erfordert. Dabei muss stets gewährleistet werden, dass die relevanten Umweltbedingungen durch die Kriterien vollständig identifiziert und für das Verfahren zweckmäßig abgedeckt werden.

Bei der quantitativen Erfassbarkeit der Kriterien kann es ebenfalls oftmals zu Schwierigkeiten kommen, da es Bereiche im Unternehmen gibt, die nur sehr schwer messbar sind. Dies ist besonders dann der Fall, wenn so genannte weiche Faktoren, wie zum Beispiel die Unternehmenskultur, berücksichtigt werden sollen. Hierin bestehen weitere Entwicklungsmöglichkeiten für das Verfahren, in dem zum einen weiche Kriterien einfließen und zum anderen deren operative Erfassbarkeit sichergestellt wird. Zudem kann bei den internen Kriterien der Informationssystemarchitektur überlegt werden, mit welchen einzelnen Maßnahmen diese in die gewünschte Richtung beeinflusst werden können. Durch diese Vorgehensweise kann ein umfassendes, ganzheitliches Verfahren aufgestellt werden, welches ein effektives und effizientes Werkzeug zur Bewältigung der dynamischen Unternehmensumwelt darstellt. 


\section{Literatur}

[AnGr04] Andresen, K., Gronau, N.: Der Faktor Wandlungsfähigkeit bei der Planung neuer Fabriken - Ein Marktüberblick von Unternehmensberatungen im Bereich Fabrikplanung. Industrie Management 20 (2004) Nr. 4, S. 34-40

[AiDo04] Aier, S., Dogan, T.: Nachhaltigkeit als Gestaltungsziel von Unterneh-mensarchitekturen; in: Aier, S., Schönherr, M. (Hrsg): Enterprise Application Integration - Serviceorientierung und nachhaltige Architekturen, Berlin 2004: Gito. Reihe: Enterprise Architecture, Band 2, noch nicht erschienen.

[Bas ${ }^{+}$03] Bass, L., Clements, P., Kazman, R.: Software Architecture in Practice; 2. Auflage, Boston 2003

[DoLa02] Doppler, K.: Lauterburg, C.: Den Unternehmenswandel gestalten; 10 Auflage, Frankfurt 2002

[DrWe84] Draft, R.L., Weick, K.E.: Toward a Model of Organizations as Interpretation Systems, in: Academy of Management Review 9 (1984), S. 284-295.

[Dunn03] Dunn, B.: A Manager's Guide to Web Services. In: EAI Journal January 2003, S. 14-17.

[EvLa ${ }^{+}$02] Eversheim, W., Lange-Stalinski, T., Redelstab, P.: Wandlungsfähigkeit durch mobile Fabriken, Werkstattstechnik online, Jahrgang 92, Nr. 4, Düsseldorf, 2002, S. 169-170.

[FaNa86] Fahey, L., Narayanan, V.K.: Macroenvironmental Analysis for Strategic Management, St. Paul, MN: West, 1986.

[FrSt02] Frese, E., Stöber, H. (Hrsg). E-Organisation. Wiesbaden 2002

[Gel198] Gell-Mann, M.: Das Quark und der Jaguar - Vom Einfachen zum Komplexen; München 1998

[Gold ${ }^{+}$95] Goldman, S. L., Nagel, R. N., Preiss, K.: Agile competitors and virtual organizations: strategies for enriching the customer; New York 1995

[Gron03] Gronau, N.: Wandlungsfähige Informationssystemarchitekturen - Nachhaltigkeit bei organisatorischem Wandel; Berlin 2003

[Gümb04] Gümbel, H.: Semiramis - The Native Next-Generation Business Software, 4.5. Vers., A White Paper, 2004.

[Hahn03] Hahn, A,: Web Services for Semantic Model Integration in Concurrent Engineering. In (Jardim-Goncalves, R.; Cha, J.; Steiger-Garcao, A. Hrsg.): Concurrent Engineering: Enhanced Interoperable Systems, A. A. Balkema , 2003, S. 557-563

[Hall77] Hall, R.H.: Organizations. Structure and Process, Prentice-Hall: Englewood Cliffs, New Jersey, 1977

[Hart02] Hartmann, M., (Hrsg.): DaWa aktuell Nr.5, Informationsblatt zum DaWa - Projekt, DaWa aktuell Nr. 4, Leonberg 2002, 
http://www.dawa-online.de/infoplattform/veroeffentlichungen/dawa_aktuell_nr4.pdf, Zugriff am 12.06.2004.

[HBK03] Hahn, A.; Benger, A.; Kern, E-M.: Peer-to-Peer Process Integration in Virtual Engineering Origanizations. In (Camarinha-Matos, J.; Afsarmanesh, H. Hrsg.): Processes and Foundations for Virtual Organizations, Kluwer Academic Publishers, 2003

[HeHo04] Heger, L.C., Holzer, H.: Wie wird der Wandel wirtschaftlich? In Industrie Management 20, Nr. 3, 2004: S. 12-16

[Hern02] Hernández, R.: Systematik der Wandlungsfähigkeit in der Fabrikplanung. In: Fortschritt-Berichte VDI, Reihe 16, Nr. 149. Düsseldorf 2002

[KiWa03] Kieser, A., Walgenbach, P.: Organisation; 4. Auflage, Stuttgart 2003

[Knof92] Knof, H.-L.: CIM und organisatorische Flexibilität; München 1992 (zugl. Dissertation, Universität Bochum, 1991).

[Krcm90] Krcmar, H.: Bedeutung und Ziele von Informationssystem-Architekturen; Wirtschaftsinformatik, 32 Jg. (1990), Nr. 5, S. 395-402.

[Krcm03] Krcmar, Helmut: Infomationsmanagement. 3. Auflage Berlin Heidelberg New York, 2003

[Krüg98] Krüger, W.: Management permanenten Wandels; in: Glaser, H., Schröder, E.F., Werder, A. V.(Hrsg): Organisation im Wandel der Märkte, Wiesbaden 1998, S. $227-$ 249.

[Laud02] Laudon, K. C., Laudon, J. P. (Hrsg). Management Information Systems; 7. Auflage. New Jersey 2002

[LeHu98] Lewin, A. Y., Hunter, S.D.; Information Technology \& Organizational Design: A Longitudinal Study of Information Technology Implementations in the U.S. Retailing Industry, 1980-1996; in: Glaser, H., Schröder, E.F., Werder, A. V.(Hrsg): Organisation im Wandel der Märkte, Wiesbaden 1998, S. 251-286.

[MaVa87] Maturana, H., Varela, F.: The Tree of Knowledge. Boston 1987

[MüLe03] Müller-Stewens, G., Lechner, C. (2003), Strategisches Management, 2.Aufl., Stuttgart 2003.

[NoK102] Nofen, D., Klußmann, J.: Wandlungsfähigkeit durch modulare Fabrikstrukturen, Industrie Management 18, Nr. 3, S. 49-52.

[Pfau97] Pfau, W.: Betriebliches Informationsmanagement - Flexibilisierung der Informationsinfrastruktur; Reihe: Markt- und Unternehmensentwicklung, Herausgegeben von: Picot, A., Reichwald, R., Wiesbaden 1997.

[Pico ${ }^{+}$03] Picot, A., Reichwald, R., Wiegand, R. T.: Die Grenzenlose Unternehmung - Information, Organisation und Management; 4. Auflage, Wiesbaden 2003.

[Port98] Porter, M.E.: Competitive Strategy: Techniques for Analyzing industries and competitors, The Free Press, 1998

[QuWi03] Quantz, J., Wichmann, T.; E-Business-Standards in Deutschland - Bestandsaufnahme, Probleme, Perspektiven: Berlecom Research 2003, 
http://www.bmwi.de/Redaktion/Inhalte/Downloads/e-business-standards-endbericht, Zugriff am29.06.2004.

[ReMö99] Reichwald, R., Möslein K.: Organisation - Strukturen und Gestaltung, in: Hoyos, C. Graf, Frey, D. (Hrsg.): Arbeits- und Organisationspsychologie. Ein Lehrbuch, Göttingen 1999: S. 29-49.

[Schr03] Schreyögg, G.: Organisation - Grundlagen moderner Organisationsgestaltung; 4. Auflage, Wiesbaden 2003

[Sinz02] Sinz, E. J.: Architektur von Informationssystemen; in: Rechenberg, P., Pomberger, G.: Informatik-Handbuch. 3. Auflage, München 2002.

[Spat $\left.{ }^{+} 01\right]$ Spath, D., Baumeister, M., Barrho, T. Et al.: Change Management im Wandel; Industrie Management, 17. Jg. (2001), Nr. 4, S. 9-13.

[ScKi03] Scheer, A. W., Kirchmer, M.: Change Management - der Schlüssel zu Business Process Excellence; in: Scheer, A.-W., Abolhassan, F., Jost, W. et al. (Hrsg): Change Management im Unternehmen: Prozessveränderungen erfolgreich managen, Berlin, New York 2003, S. 1-14.

[StPe04] Stannat A., Petri, C.: Trends in der Unternehmens-IT, Mittelfristige Entwicklungen in der Informationstechnologie und in IT Organisationen aus Sicht von Unternehmen und öffentlichen Organisationen; Informatik Spektrum, Heidelberg, 2004, S. $227-$ 237.

[StSc00] Steinmann, H., Schreyögg, G.: Management, 5. Aufl., Wiesbaden, 2000.

[Thom67] Thompson, J.P.: Organizations in Action, New York 1967.

[Wall96] Wall, F.: Organisation und betriebliche Informationssysteme - Elemente einer Konstruktionslehre; Wiesbaden 1996 (zugl. Habilitation, Universität Hamburg, 1996).

[Warn93] Warnecke, H.-J.: Revolution der Unternehmenskultur. Das Fraktale Unternehmen. 2. Auflage Berlin Heidelberg New York 1993

[West02] Westkämper, E. et. Al., : Ansätze zur Wandlungsfähigkeit von Produktionsunternehmen. Ein Bezugsrahmen für die Unternehmensentwicklung im turbulenten Umfeld, wt: Werkstattstechnik, Jahrgang 90, Nr. 1/2, Düsseldorf, 2004, S. 22-26.

[WiEr ${ }^{+}$03] Wirth, S., Enderlein, H., Hildebrand, T.: PLUG+PRODUCE. Ein Modularkonzept zur effizienten Gestaltung wandelbarer Produktions- und Organisationsstrukturen durch Vernetzung, Industrie Management 17, Nr. 5, S. 67-70. 